\title{
Nondispersive Fermi Arcs and the Absence of Charge Ordering in the Pseudogap Phase of $\mathrm{Bi}_{2} \mathrm{Sr}_{2} \mathrm{CaCu}_{2} \mathrm{O}_{8+\delta}$
}

\author{
U. Chatterjee, ${ }^{1,2}$ M. Shi, ${ }^{1,3}$ A. Kaminski, ${ }^{4}$ A. Kanigel, ${ }^{1}$ H. M. Fretwell, ${ }^{4}$ K. Terashima, ${ }^{5}$ T. Takahashi, ${ }^{5}$ S. Rosenkranz, ${ }^{2}$ \\ Z.Z. Li, ${ }^{6}$ H. Raffy, ${ }^{6}$ A. Santander-Syro, ${ }^{6}$ K. Kadowaki, ${ }^{7}$ M. R. Norman, ${ }^{2}$ M. Randeria, ${ }^{8}$ and J. C. Campuzano ${ }^{1,2}$ \\ ${ }^{1}$ Department of Physics, University of Illinois at Chicago, Chicago, Illinois 60607, USA \\ ${ }^{2}$ Materials Science Division, Argonne National Laboratory, Argonne, Illinois 60439, USA \\ ${ }^{3}$ Swiss Light Source, Paul Scherrer Institut, CH-5232 Villigen, Switzerland \\ ${ }^{4}$ Ames Laboratory and Department of Physics and Astronomy, Iowa State University, Ames, Iowa 50011, USA \\ ${ }^{5}$ Department of Physics, Tohoku University, 980-8578 Sendai, Japan \\ ${ }^{6}$ Laboratorie de Physique des Solides, Universite Paris-Sud, 91405 Orsay Cedex, France \\ ${ }^{7}$ Institute of Materials Science, University of Tsukuba, Ibaraki 305-3573, Japan \\ ${ }^{8}$ Department of Physics, Ohio State University, Columbus, Ohio 43210, USA
}

(Received 10 May 2005; published 16 March 2006)

\begin{abstract}
The autocorrelation of angle resolved photoemission data from the high temperature superconductor $\mathrm{Bi}_{2} \mathrm{Sr}_{2} \mathrm{CaCu}_{2} \mathrm{O}_{8+\delta}$ shows distinct peaks in momentum space which disperse with binding energy in the superconducting state, but not in the pseudogap phase. Although it is tempting to attribute a nondispersive behavior in momentum space to charge ordering, a deconstruction of the autocorrelation reveals that the nondispersive peaks arise from the tips of the Fermi arcs, which themselves do not change with binding energy.
\end{abstract}

DOI: 10.1103/PhysRevLett.96.107006

PACS numbers: 74.25.Jb, 74.72.Hs, 79.60.Bm

A central issue in condensed matter physics is the competition between different order parameters. This competition is most dramatic in the transition metal oxides which show a rich variety of insulating, conducting, magnetic, and superconducting phases. In the case of the high temperature cuprate superconductors, the situation is particularly interesting, with a mysterious pseudogap phase lying between the insulating state at zero doping and the $d$-wave superconducting state. It has been suggested that a hard-todetect order might underlie the pseudogap phenomenon, and recent Fourier transform scanning tunneling spectroscopy (FT-STS) experiments [1-3] on $\mathrm{Bi}_{2} \mathrm{Sr}_{2} \mathrm{CaCu}_{2} \mathrm{O}_{8+\delta}$ have been interpreted in terms of local charge ordering in the pseudogap phase.

We address this question using a novel autocorrelation analysis of angle resolved photoemission spectroscopy (ARPES) data. We first demonstrate that the highly dispersive vectors observed in the superconducting state FTSTS originate from the high density of states at the end of the constant energy contours ("bananas") $[1,2,4]$. Next, we show that the locus of low energy excitations in the pseudogap state, the "Fermi arcs" [5], are essentially independent of energy [6]. This remarkable fact is shown to lead to nondispersing peaks in the ARPES autocorrelation, in detailed agreement with FT-STS [3]. We do not find any evidence in ARPES of charge ordering in the pseudogap phase, nor do we need to invoke it to understand the dispersionless autocorrelation peaks.

We analyze ARPES data by looking at the autocorrelation $C(\mathbf{q}, \omega)=\sum_{\mathbf{k}} I(\mathbf{k}+\mathbf{q}, \omega) I(\mathbf{k}, \omega)$ of the ARPES intensities $I(\mathbf{k}, \omega)$, i.e., the correlation of intensities at two different momenta, separated by a momentum transfer $\mathbf{q}$, at a fixed energy. We shall show that the autocorrelation, which is effectively the momentum-resolved joint density of states, gives us important new insights into both the superconducting and pseudogap states. $C(\mathbf{q}, \omega)$ exhibits discrete spots in $\mathbf{q}$ space which can be directly related to recent FT-STS results [1-3]. We note that the ARPES autocorrelation does not require any theoretical modeling for its interpretation and the observed $\mathbf{q}$-space pattern can be directly interpreted from the measured ARPES intensity. By restricting the autocorrelations over specific regions of the intensity maps, we can unambiguously determine the origin of those spots.

The $\mathbf{k}$ sum in the definition of the autocorrelation can be handled in one of two ways. In the first method (A) we take both $\mathbf{k}$ and $\mathbf{k}+\mathbf{q}$ within the first Brillouin zone. Hence the number of data points in the sum contributing to a given $\mathbf{q}$ decreases with increasing $|\mathbf{q}|$, and we compensate for this by normalizing with the number of points used in the sum. The second method (B) is to impose periodic boundary conditions in $\mathbf{k}$ (i.e., a repeated zone scheme) although we do not focus on these results here for reasons discussed later.

We show data on single crystals (grown using the floating zone method) and thin films (made by rf sputtering technique) of $\mathrm{Bi}_{2} \mathrm{Sr}_{2} \mathrm{CaCu}_{2} \mathrm{O}_{8+\delta}$. The samples were mounted with the $\mathrm{Cu}-\mathrm{O}$ bond direction parallel to the photon polarization, and cleaved in situ at pressures below our measurement limit of $2 \times 10^{-11}$ Torr. The $22 \mathrm{eV}$ incident photons were not aligned along a mirror plane to avoid strong selection rules. Measurements were carried out at the Synchrotron Radiation Center in Madison, Wisconsin, on the $\mathrm{U} 1$ undulator beam line, at $2 \mathrm{meV}$ 
intervals, using a Scienta SES 2002 electron analyzer. The energy resolution was $15 \mathrm{meV}$ and the $\mathbf{k}$ resolution was $0.0036 \AA^{-1}$ for the thin films, and $0.01 \AA^{-1}$ for the single crystals along the multiplexing direction of the detector, and $\sim 0.02 \AA^{-1}$ along the perpendicular direction in both cases. The $\mathbf{k}$ resolutions along the detector cut are comparable to or better than that of FT-STS, which for the largest field of view studied is about $0.01 \AA^{-1}$.

The data were taken in the $Y$ quadrant to minimize superlattice effects. The raw data covered the full quadrant (for Fig. 2) and one-half of it (for Figs. 1 and 3). The intensity map in the whole Brillouin zone was reconstructed by reflections, using interpolation to a uniform grid. The large background emission in ARPES reduces the contrast in the autocorrelation maps. We deal with this by simply zeroing out the intensity once it falls below a given threshold value, which is $20 \%$ of the maximum intensity (for a given $\omega$ ) for the superconducting phase and $38 \%$ in the pseudogap state. We have checked the choice of threshold does not affect anything other than the contrast in the autocorrelations. Finally, the spectra were normalized at high binding energy $(500 \mathrm{meV})$ to minimize the effect of the dipole matrix elements.

In Figs. 1(a) and 1(b) we show the ARPES intensity maps, $I(\mathbf{k}, \omega)$, as a function of the momentum $\mathbf{k}$ in the first zone, for two different choices of the binding energy $\omega$. These data are obtained for an optimally doped single crystal sample $\left(T_{c}=90 \mathrm{~K}\right)$ in the superconducting state $(T=40 \mathrm{~K})$. (We obtain similar results for the thin film samples, which are not shown here for brevity.) The characteristic "banana" shapes of the constant energy contours due to the $d$-wave energy gap can be clearly seen. We note two very important features: (i) the extent of the bananas increases with increasing binding energy, and (ii) the highest intensity points are located at the tips of the banana. In the right panels [Figs. 1(c) and 1(d)], we plot the autocorrelation of the ARPES intensities which exhibits discrete spots in q space that disperse with energy. We see that the spots in the autocorrelation in Figs. 1(c) and 1(d) can be directly correlated with those wave vectors $\mathbf{q}_{i}$ that connect the tips of the bananas in the intensity map of Figs. 1(a) and 1(b), respectively. We also see that each of these $\mathbf{q}_{i}$ vectors disperses as a function of the binding energy $\omega$. This is shown in detail in Fig. 1(e) for the vectors $\mathbf{q}_{1}$ and $\mathbf{q}_{5}$ along the bond direction, and in Fig. 1(f) for the vectors $\mathbf{q}_{3}$ and $\mathbf{q}_{7}$ along the zone diagonal. The dispersion of the autocorrelation spots follows the evolution of the tips of the bananas with $\omega$.

Remarkably, these $\mathbf{q}_{i}$ vectors are in quantitative agreement with those obtained by the Davis group [1,2], and therefore confirm that the "octet" model [4] used to explain the FT-STS results does indeed describe the regions of highest joint density of states. It is surprising that FTSTS shows the same q vectors, given that the formula used to theoretically model the FT-STS data, $\left[\operatorname{Im} \sum_{\mathbf{k}} \hat{G}(\mathbf{k}+\right.$
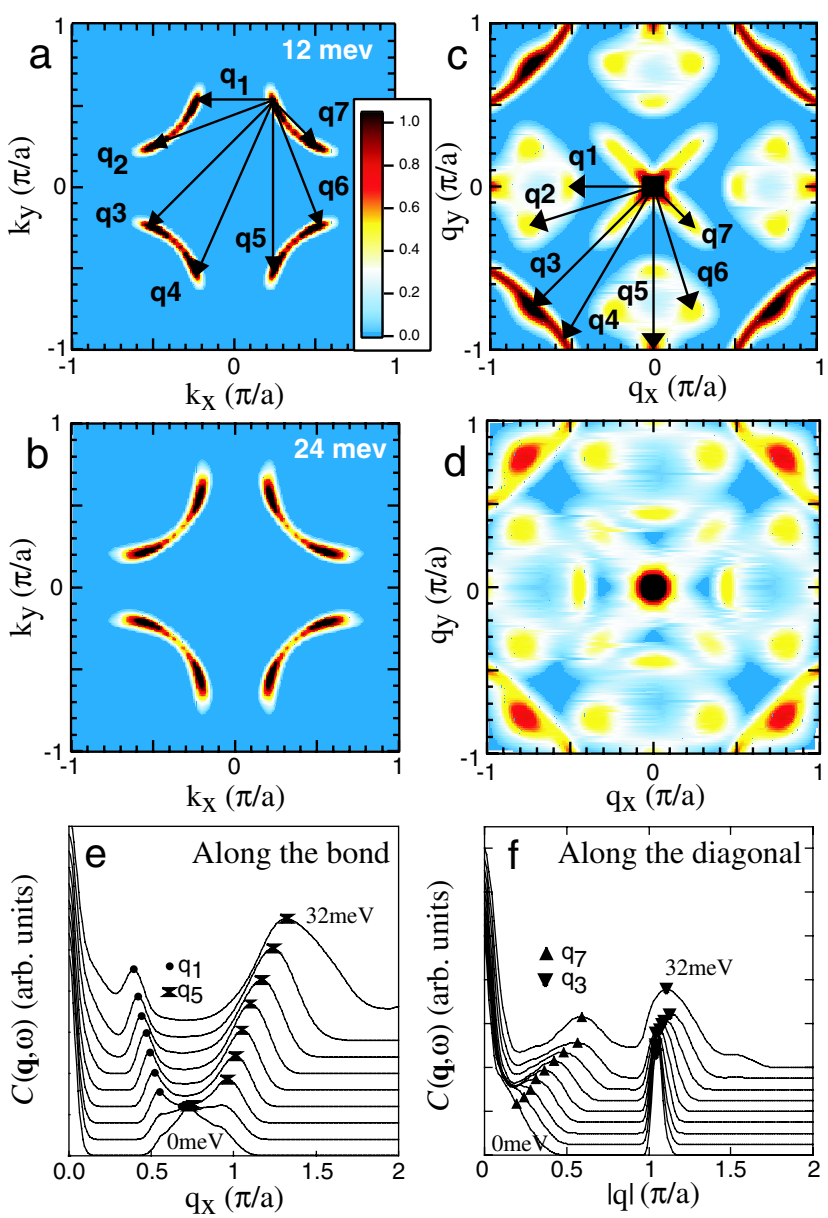

FIG. 1 (color online). Spectral intensities and their autocorrelations in the superconducting state $(T=40 \mathrm{~K})$ for an optimally doped single crystal sample. Intensity at a binding energy of (a) $12 \mathrm{meV}$ and (b) $24 \mathrm{meV}$ in the first zone. (c), (d) Autocorrelation of the data in (a), (b), with vectors in (c) indicating local maxima in the autocorrelation which correspond to equivalent vectors shown in (a). The color scales in these plots are in arbitrary units. (e) Autocorrelation of the data as a function of binding energy along the $(q, 0)$ direction. The lowest curve is at zero energy, with the binding energy increasing by $4 \mathrm{meV}$ for each subsequent curve (the curves are displaced vertically for clarity). (f) Same as (e), but along the $(q, q)$ direction. For both directions, strongly dispersing peaks are seen.

$\mathbf{q}, \omega) \hat{T}(\mathbf{k}+\mathbf{q}, \mathbf{k}, \omega) \hat{G}(\mathbf{k}, \omega)]_{11} \quad$ (where $\hat{G}$ are Nambu Greens functions), that describes the scattering of electrons from the state $\mathbf{k}$ to the state $\mathbf{k}+\mathbf{q}$ by the scattering matrix $\hat{T}$, is not mathematically equivalent to the simple autocorrelation used in our analysis.

When we used periodic boundary conditions (method B) for our autocorrelation sum we observed additional umklapp peaks, e.g., at $(2 \pi / a, 0)-\mathbf{q}_{1}$. Such peaks are not visible in the FT-STS data and are implicitly ignored in the "octet model" [4]. Thus we get good agreement with the superconducting state FT-STS data only if we use method 
A (see above) as opposed to B. We then continue to use the same procedure (A) in the remainder of this Letter.

We now turn to the less understood pseudogap phase. This phase is characterized by Fermi arcs (gapless segments in $\mathbf{k}$ space) above $T_{c}$ [5]. In Fig. 2 we show data similar to Fig. 1, but now in the pseudogap phase $(T=$ $90 \mathrm{~K})$ of an underdoped thin film sample $\left(T_{c}=76 \mathrm{~K}\right)$. There are two significant features of the intensity plots in Figs. 2(a) and 2(b). (i) The Fermi arcs do not significantly change with binding energy, and (ii) the intensity along the arc is fairly uniform and does not peak at the tips. Both of these features are in sharp contrast to the $d$-wave superconducting state bananas. The autocorrelation of these data along the bond direction is shown in Fig. 2(c) and exhibits clearly identifiable peaks near $\mathbf{q}^{*}=(0.4 \pi / a, 0)$. Further, as shown in Fig. 2(c), these autocorrelation peaks show very little dispersion, in marked contrast to the supercon-
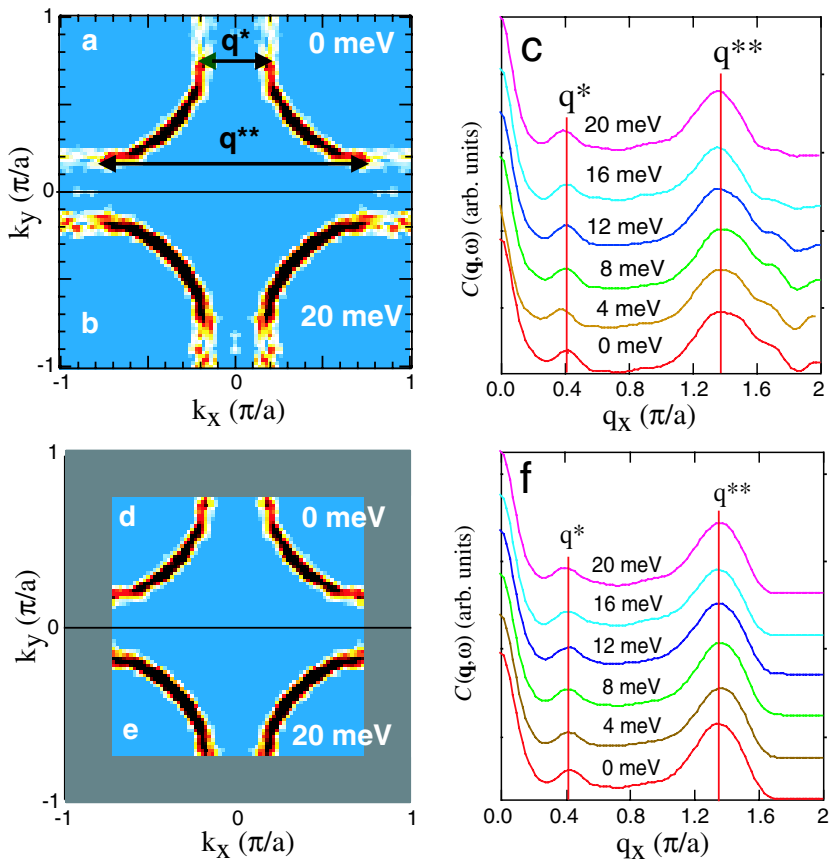

FIG. 2 (color online). Fermi arcs in the pseudogap state and their autocorrelations. Spectral intensity in the top half of the first zone at (a) $0 \mathrm{meV}$ and (b) $20 \mathrm{meV}$ for an underdoped thin film sample $\left(T_{c}=76 \mathrm{~K}\right)$ at $T=90 \mathrm{~K}$. One can see that the length of the arcs remains unchanged over the energy scale of the pseudogap. (c) Autocorrelation as a function of binding energy along the $(q, 0)$ direction. Note the dispersionless nature of the peaks labeled $\mathbf{q}^{*}$ and $\mathbf{q}^{* *}$. (d) Spectral intensity as in (a), but with regions beyond the Fermi arcs cut off from the data. (e) Spectral intensity as in (b), but with the data cut off as in (d). (f) Autocorrelation with data beyond the Fermi arcs cut off, as shown for two energies in (d) and (e). Note that the results are unchanged from (c) for the $\mathbf{q}^{*}$ and $\mathbf{q}^{* *}$ peaks, indicating that the peaks in the autocorrelation originate from the ends of the arcs, and not from the pseudogapped sections of the Fermi surface. ducting state results of Fig. 1. Both the wave vector and its lack of dispersion are consistent with FT-STS results in the pseudogap phase [3]. We have obtained similar results for both thin film (Fig. 2) and single crystal (Fig. 3) samples at different temperatures and dopings, and therefore conclude that these results are general to the pseudogap phase. At higher energies, additional features appear in the autocorrelations from the pseudogapped regions of the zone.

We now use our intensity data to understand the origin of the dispersionless autocorrelation peaks seen in the pseudogap phase. We find that these peaks correspond to vectors connecting the ends of the Fermi arcs [5], where there is a sudden onset of the pseudogap. This onset is sufficiently steep so the Fermi arcs have nearly constant length for all binding energies smaller than the pseudogap value. This gives rise to the dispersionless nature of the autocorrelation vectors. We arrive at this conclusion by truncating the intensity plots to include only the Fermi arcs, as shown for 0 and $20 \mathrm{meV}$ in Figs. 2(d) and 2(e). We find that the autocorrelation of these data, shown in Fig. 2(f), yields the
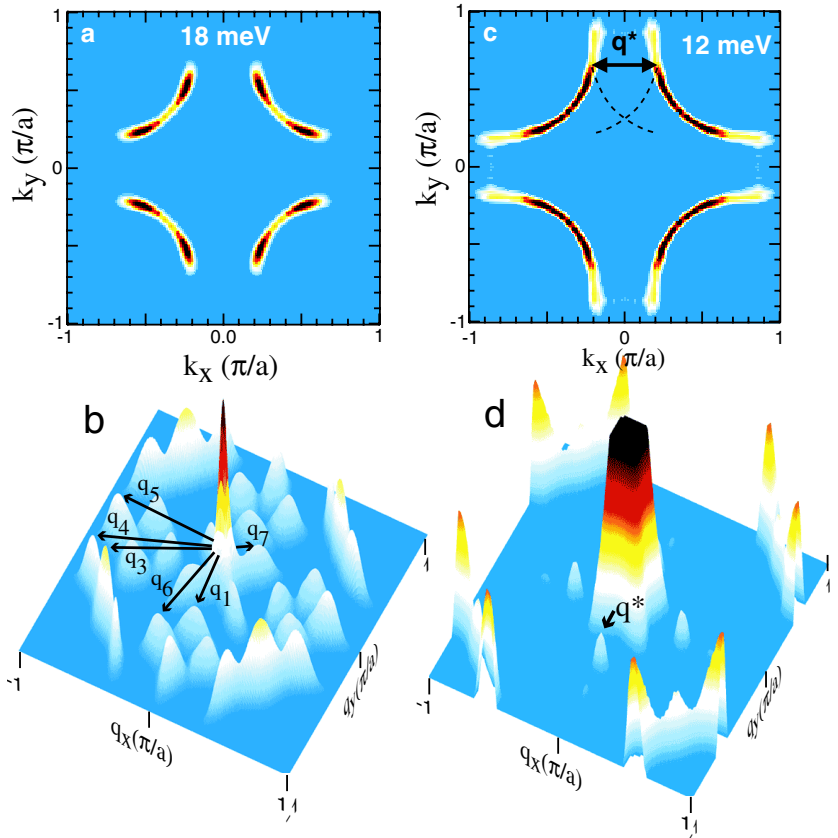

FIG. 3 (color online). Comparison of autocorrelations of similar intensity maps in the superconducting and pseudogap phases of a single crystal sample $\left(T_{c}=90 \mathrm{~K}\right)$. (a) Intensity map in the superconducting state at $T=40 \mathrm{~K}$ at $18 \mathrm{meV}$. (b) Surface plot of the autocorrelation function from (a). (c) Comparison of an equal length Fermi arc in the pseudogap state $(T=140 \mathrm{~K})$ at $12 \mathrm{meV}$ to the superconducting banana in (a) obtained from the same sample. The dashed lines in (c) represent "shadow bands" (arcs displaced by $\mathbf{q}^{*}$ ) expected from charge ordering at a wave vector $\mathbf{q}^{*}$. (d) Surface plot of the autocorrelation function from (c). Note the significant differences between the two autocorrelation maps - (b), (d) — despite the similarities of the intensity maps (a), (c). 
same result as in Fig. 2(c). Therefore, we conclude that $\mathbf{q}^{*}$ connects the ends of the Fermi arcs, rather than the parallel sections near the zone faces, whose faint traces can be seen in Figs. 2(a) and 2(b). In any case the intensity in the straight sections is much too weak at low energies to produce the $\mathbf{q}^{*}$ peak.

The existence of $\mathbf{q}^{*}$ spanning the shorter distance between arc tips implies that a $\mathbf{q}^{* *}$ spanning the longer distance between arc tips along the bond direction must also be present. This is indeed seen in our autocorrelation plot in Fig. 2(c). The latter $\mathbf{q}^{* *}$ was not reported in Ref. [3], but has been seen recently [7]. We also find additional peaks along the diagonal direction, as shown in the autocorrelation surface plot in Fig. 3(d). These originate from vectors analogous to $\mathbf{q}_{3}$ and $\mathbf{q}_{4}$ in the superconducting state, which span the ends of the arcs along or near the diagonal directions. We speculate that the differences with FT-STS data arise because large wave-vector signals are suppressed by the spatial extent of the STM tip and matrix elements suppress tunneling into states near the zone diagonal. We also note that in the superconducting FT-STS data the intensity of the $\mathbf{q}_{3}$ and $\mathbf{q}_{4}$ peaks drops sharply with reduced doping [8].

While the octet models and variants have been proposed to explain the FT-STS observations in terms of scattering of electrons by impurities in the superconducting state $[1,2,4,9,10]$, these models do not describe the pseudogap data [11]. On the other hand, an autocorrelation analysis of ARPES data does not require a model for its interpretation, which leads us to our most significant finding: all features, in both the superconducting and pseudogap cases, have a common origin in spite of the dramatic differences between the autocorrelation maps in the superconducting [Fig. 3(b)] and pseudogap [Fig. 3(d)] states. The peaks in the autocorrelation are always associated with the momentum-dependent high joint density of states. In the superconducting state, it is the high intensity at the ends of the bananas which lead to the autocorrelation peaks. In the pseudogap state there are many $k$ points with reasonably high intensity near the tip of the arc, separated by nearly the same wave vector, which boosts the autocorrelation and leads to the $\mathbf{q}^{*}$ peak.

The dispersionless $\mathbf{q}^{*}$ spots in the pseudogap phase have previously been interpreted as evidence for charge ordering $[3,12,13]$. We offer a different interpretation here. Charge ordering should lead to "shadow" bands, that is, images of the bananas or "arcs" translated by the presumed ordering wave vector $\mathbf{q}^{*}$ as indicated by the dashed curves in Fig. 3(c). Even if the shadow features were very weak, there should have been a predicted [14] anomaly in the linewidth near the tip of the arc. We find no evidence for either shadow bands or linewidth anomalies. We can, however, simply understand the dispersionless $\mathbf{q}^{*}$ spots in terms of the constant length Fermi arcs, without invoking charge ordering. It is an interesting open question whether or not the low temperature FT-STS data on highly underdoped samples of a different material, $\mathrm{Ca}_{2-x} \mathrm{Na}_{x} \mathrm{CuO}_{2} \mathrm{Cl}_{2}$ [15], can also be understood within this picture.

In summary, we use ARPES autocorrelations to gain insight into the superconducting and pseudogap phases of high $T_{c}$ cuprates and make direct connection with FT-STS data. The ARPES autocorrelation peaks arise from enhanced joint density of states both above and below $T_{c}$. In the superconducting state, the strongly dispersing peaks are at $\mathbf{q}$ vectors which connect points of large density of states at the ends of the constant energy contours (bananas). In the pseudogap state, we find nondispersive peaks at q's joining the tips of the Fermi arcs. We find no direct evidence for charge ordering from our data, but can nevertheless directly relate the lack of dispersion of autocorrelation peaks in the pseudogap state to the remarkable energy independence of the Fermi arcs.

We acknowledge useful discussions with J.C. Davis and A. Yazdani. This work was supported by NSF DMR 0305253, the U.S. DOE, Office of Science, under Contracts No. W-31-109-ENG-38 (ANL) and No. W-7405Eng-82 (Ames), and the MEXT of Japan. The Synchrotron Radiation Center is supported by NSF DMR-0084402.

[1] J. E. Hoffman et al., Science 297, 1148 (2002).

[2] K. McElroy et al., Nature (London) 422, 592 (2003).

[3] M. Vershinin et al., Science 303, 1995 (2004).

[4] Q.-H. Wang and D.H. Lee, Phys. Rev. B 67, 020511 (2003).

[5] M. R. Norman et al., Nature (London) 392, 157 (1998); Phys. Rev. B 57, R11093 (1998).

[6] We use the notation "Fermi arcs" for the constant energy features, even for nonzero binding energy.

[7] A. Yazdani (private communication).

[8] K. McElroy et al., cond-mat/0404005.

[9] L. Capriotti, D. J. Scalapino, and R. D. Sedgewick, Phys. Rev. B 68, 014508 (2003).

[10] R. S. Markiewicz, Phys. Rev. B 69, 214517 (2004).

[11] T. Pereg-Barnea and M. Franz, Phys. Rev. B 68, 180506 (2003).

[12] S. A. Kivelson et al., Rev. Mod. Phys. 75, 1201 (2003).

[13] A. Polkovnikov, M. Vojta, and S. Sachdev, Phys. Rev. B 65, 220509 (2002).

[14] D. Podolsky et al., Phys. Rev. B 67, 094514 (2003).

[15] T. Hanaguri et al., Nature (London) 430, 1001 (2004). 